\title{
Stroma secreted IL6 selects for "stem-like" population and alters pancreatic tumor microenvironment by reprogramming metabolic pathways
}

\author{
Kousik Kesh ${ }^{1,2}$, Vanessa T. Garrido ${ }^{1,2}$, Austin Dosch ${ }^{1,2}$, Brittany Durden ${ }^{1,2}$, Vineet K. Gupta ${ }^{1,2}$, Nikita S. Sharma (1) ${ }^{1,2}$, \\ Michael Lyle ${ }^{3}$, Nagaraj Nagathihalli ${ }^{1,2}$, Nipun Merchant ${ }^{1,2}$, Ashok Saluja ${ }^{1,2}$ and Sulagna Banerjee $\mathbb{E}^{1,2}$
}

\begin{abstract}
Pancreatic adenocarcinoma is a devastating disease with an abysmal survival rate of $9 \%$. A robust fibro-inflammatory and desmoplastic stroma, characteristic of pancreatic cancer, contribute to the challenges in developing viable therapeutic strategies in this disease. Apart from constricting blood vessels and preventing efficient drug delivery to the tumor, the stroma also contributes to the aggressive biology of cancer along with its immune-evasive microenvironment. In this study, we show that in pancreatic tumors, the developing stroma increases tumor initiation frequency in pancreatic cancer cells in vivo by enriching for CD133 + aggressive "stem-like" cells. Additionally, the stromal fibroblasts secrete IL6 as the major cytokine, increases glycolytic flux in the pancreatic tumor cells, and increases lactate efflux in the microenvironment via activation of the STAT signaling pathway. We also show that the secreted lactate favors activation of M2 macrophages in the tumor microenvironment, which excludes CD8 + T cells in the tumor. Our data additionally confirms that the treatment of pancreatic tumors with anti-IL6 antibody results in tumor regression as well as decreased CD133 + population within the tumor. Furthermore, inhibiting the lactate efflux in the microenvironment reduces M2 macrophages, and makes pancreatic tumors more responsive to anti-PD1 therapy. This suggests that stromal IL6 driven metabolic reprogramming plays a significant role in the development of an immune-evasive microenvironment. In conclusion, our study shows that targeting the metabolic pathways affected by stromal IL6 can make pancreatic tumors amenable to checkpoint inhibitor therapy.
\end{abstract}

\section{Introduction}

Pancreatic cancer has emerged as one of the most aggressive malignancies with a dismal 5-year survival rate of a mere $9 \%{ }^{1}$. Aggressive biology, manifested by a high rate of tumor recurrence and resistance to therapy, contributes to poor prognosis in this disease. The pancreatic tumor microenvironment is characterized by the presence

Correspondence: Sulagna Banerjee (Sulagna.banerjee@med.miami.edu) 'Department of Surgery, Miller School of Medicine, University of Miami, Miami, $\mathrm{FL}$, USA

${ }^{2}$ Sylvester Comprehensive Cancer Center, Miami, FL, USA

Full list of author information is available at the end of the article

Edited by C. Munoz-Pinedo of a robust, fibro-inflammatory stroma that is largely immunosuppressive. During tumor progression, this microenvironment develops anatomically distinct "niches", leading to enrichment of a small population of "stem-like" cells that have a distinct survival advantage over the bulk of the tumor cells. This population contributes to the aggressive biology of the disease $\mathrm{e}^{2-4}$. Our previous studies show that this aggressive population has increased selfrenewal properties (contributing to tumor recurrence), increased invasiveness, and ability to evade adverse chemotherapeutic assault on the tumor and is represented by the expression of CD133 on their surface ${ }^{5,6}$. Hypoxic regions, nutritional stress, and chemotherapeutic agents 
enrich for this treatment-refractory population within the tumor by converting non-aggressive CD133- cells to an aggressive CD133+ population ${ }^{7-10}$. This dynamic interconversion of a non-aggressive population to an aggressive one contributes to a major challenge while developing effective therapy.

The pro-metastatic and pro-tumorigenic role of the pancreatic cancer stroma is well-studied ${ }^{11-14}$. In liver and lung cancer, the stromal cells have been reported to reprogram the tumor cells ${ }^{15}$. However, the extent to which the stromal components affect the properties of the tumor is an ongoing area of research. Stroma secreted cytokines like IL6 has been implicated as one of the major inflammatory mediators in pancreatic cancer ${ }^{16,17}$. Independent of cytokines, stroma-derived metabolites have been shown to promote autophagy in tumor cells and contribute to tumor progression ${ }^{18-20}$. While the coculture of stromal cells with tumor cells is known to enrich for tumor-initiating cells or TIC in other cancers $^{21-23}$, whether they promote the enrichment of CD133 + TIC population in pancreatic cancer is not known. Metabolic reprogramming in a tumor cell plays a crucial role for it to develop into an aggressive state that has a high recurrence potential ${ }^{24}$. Recent research shows that the hypoxic microenvironment enriches for $\mathrm{CD} 133$ + population within a tumor ${ }^{7,25,26}$. Classically, the hypoxic microenvironment alters cellular metabolism, promoting glycolysis and suppressing mitochondrial respiration. This results in a lower accumulation of ROS in these cells upon treatment with chemotherapy, eventually protecting these cells from apoptosis ${ }^{8}$. Literature shows that the altered metabolism in the tumor cell has a significant influence on the tumor microenvironment affecting polarization of macrophages to a "pro-tumorigenic" M2 phenotype that in turn can exclude cytotoxic $\mathrm{T}$ cells from entering the tumor, thereby contributing to the immune-evasive microenvironment ${ }^{27,28}$. However, whether this metabolic reprogramming drives immune suppression in pancreatic cancer is not known. Our recently published studies have shown that tumor intrinsic metabolic pathways like the hexosamine biosynthesis pathway can be targeted to modulate the microenvironment and thus sensitize pancreatic cancer cells to checkpoint inhibitor therapy ${ }^{29}$. Thus, metabolic inhibitors are being evaluated to sensitize normally immune-evasive pancreatic tumors to checkpoint inhibitor therapy.

In this study, we show that in pancreatic tumors, the developing stroma increases tumor initiation frequency in pancreatic cancer cells in vivo by enriching for CD133+ aggressive "stem-like" cells. Further, the stromal fibroblasts secrete IL6 as the major cytokine, which in turn, increases glycolysis in the pancreatic tumor cells, leading to increased lactate efflux in the microenvironment. We also show that the secreted lactate as a result of metabolic reprogramming in response to stromal IL6 remodels the tumor immune microenvironment to make it immune evasive, by favoring M2 macrophages in the microenvironment. We show that the treatment of pancreatic tumors with anti-IL6 antibody results in tumor regression as well as decreased CD133 + population within the tumor. Similarly, inhibiting the lactate efflux in the microenvironment decreases M2 macrophages and makes notoriously immune-evasive pancreatic tumors more responsive to anti-PD1 therapy.

\section{Materials and methods}

\section{Cell culture, treatment, and silencing}

MIAPaCa-2 (CRM-CRL-1420) and SU.86.86 (CRL1837) cell lines were purchased from ATCC and cultured respectively in DMEM (Hyclone) and RPMI 1640 (Hyclone) containing 10\% fetal bovine serum (FBS) with 1\% Pen Strep (Gibco). Human PSC purchased from ScienCell were maintained in steCM (ScienCell) media according to the recommended conditions. Cancerassociated fibroblasts (CAF) were isolated from KPC mice according to Dauer et al. ${ }^{30}$ and maintained in DMEM (Hyclone). All the established cell lines were used between passages 5 and 18. All cells were maintained at $37^{\circ} \mathrm{C}$ in a humidified air atmosphere with $5 \% \mathrm{CO}_{2} .70 \%$ confluent cells were used in each experiment. All cell lines were tested for mycoplasma and validated by STR profiling routinely.

Recombinant IL6 $(15 \mathrm{ng} / \mathrm{ml})$ (Abcam) was used to treat cells in culture for all experiments. For the IL6 receptor silencing, ON-TARGETplus human Il6R siRNA- SMARTpool (GE Dharmacon) was transfected using HiPerfect transfection reagent according to the manufacturer's protocols. For IL6 receptor-neutralizing experiments, cells were treated with an anti-Il6-R monoclonal antibody (B-R6, Invitrogen) $1 \mathrm{~h}$ before IL6 stimulation. Small molecules inhibitor Stattic and STF31 (Sigma) at indicated concentrations were used in various inhibition experiments. Conditioned media (CM) of tumor cells (MIA-CM) and PSC were produced using FBS-free basal media to exclude the effects of growth factors in serum for downstream experiments. Cells were initially plated in complete media for $24 \mathrm{~h}$. Upon reaching $70 \%$ confluency, cells were put in FBS-free basal media for $48 \mathrm{~h}$ to generate the CM. The resulting $\mathrm{CM}$ was centrifuged for $10 \mathrm{~min}$ at $1000 \mathrm{rpm}$ after collection and stored at $-80^{\circ} \mathrm{C}$ for no more than two months before treating the cells.

Cell proliferation in response to IL6 was measured by plating the cells and analyzing their growth using IncuCyte according to the manufacturer's instruction.

All experiments were conducted with at least three biological and technical replicates for rigor and reproducibility. 


\section{Nomenclature}

Commercially obtained activated pancreatic stellate cells of human origin are referred to as PSC throughout the text. Stellate cells isolated from the pancreas of a KPC mouse at 1 month (when the pancreas is histologically normal but harbors a KRAS and p53 mutation) is referred to as murine PSC or mPSC. CAFs are isolated from a fully developed KPC tumor. These refer to transformed mPSCs and activated fibroblasts from the host that constitute a significant part of the pancreatic tumor stroma.

\section{Boyden chamber invasion assay}

Boyden chamber invasion inserts (Corning BioCoat) were rehydrated for $2 \mathrm{~h}$ in serum-free medium at $37^{\circ} \mathrm{C}$. Cells were plated in the insert, on top of the Matrigelcoated membrane in a serum-free medium. The bottom well contained either PSC-CM or MIA-CM, serving as the attractant. After $24 \mathrm{~h}$, inserts were washed with PBS, the top of the membrane was scrubbed with a cotton swab to remove any remaining noninvaded cells, fixed in methanol, and stained with crystal violet. Invading cells were counted randomly by microscopy (magnification, $\times 20$ ).

\section{Isolation of CD133 - tumor cells}

The CD133- population was separated from MIAPaCa-2 cells using MACS separation (Miltenyi Biotech) according to the manufacturer's protocol. Cells growing in culture were scraped gently into centrifuge tubes and washed once in wash buffer (PBS, 0.5\% BSA, $2 \mathrm{mM}$ EDTA), and subsequently incubated with anti-mouse CD133 microbeads for 10 min on ice, and negatively purified for CD133- cells by MACS. The purity of separation was tested for each batch by performing a FACS analysis using anti-CD133-PE antibody AC141 (Miltenyi Biotech). Isolated cells were used for IL6 treatment and RNA analysis.

\section{Quantitative real-time PCR and PCR array}

Quantitative real-time PCR for SNAIl1, SNAIL2, SNAIL3, ZEB1, TWIST1, HSP70, HSF1, NFKB1, RelA, Survivin, OCT4, SOX2, and Nanog were performed using primers from Qiagen (QuantiTect primer assay). All data were normalized to $18 \mathrm{~S}$ (18S QuantiTect Primer Assay; Qiagen).

In another set of experiments, MIAPaCa-2 and SU.86.86 cells were plated and treated in various conditions as described above for quantitative PCR array. The human glucose metabolism array RT2 profiler PCR kit (PAHS-006ZG-4) from QIAGEN was used for RNA expression analysis, according to the manufacturer's instructions.

\section{Multiplexed cytometric bead array}

Conditioned media from MIA-PACA2, SU86.86, and PSC (MIA-CM, SU-CM, and PSC-CM, respectively) were collected from $48 \mathrm{~h}$ cell culture in FBS-free basal media. Murine Pancreatic Stellate cells (mPSC) were isolated from normal C57BL6 mice, 1-month-old KPC mice ${ }^{31}$ along with CAFs from fully grown KPC tumors. Isolated cells were grown in tissue culture dishes for 1 week. PSCs were maintained in the inactive form by plating on collagen-coated plates. The conditioned medium from these was collected. The LEGEND plex human inflammation panel 1 (BioLegend 740809) and mouse Th cytokine Panel (BioLegend 740740) were used, and the experiment was performed according to the manufacturer's instructions.

\section{Immunofluorescence and immunohistochemistry}

Tissues were deparaffinized by heating it at $56^{\circ} \mathrm{C}$ overnight and then hydrated by dipping it in xylene (15 min, two times), 100\% ethanol, 90\% ethanol, $70 \%$ ethanol (two times), and PBS 5 min each. The slides were then steamed with a $\mathrm{pH} 9$ reveal decloaker (BIOCARE Medical) for antigen retrieval, blocked in Dako serum-free blocker (Agilent technologies). Primary antibody against aSMA (Abcam), CD133 (Miltenyi), Ki67 (Invitrogen), CD206 (Abcam), CD8 (Abcam) was added overnight. Slides were washed $3 x$ in PBS, secondary antibodies (Alexafluor-conjugated) were diluted in SNIPER (BIOCARE Medical), and slides were stained for $30 \mathrm{~min}$ at room temperature. Slides were washed again $3 \times$ in PBS and mounted using Prolong Gold anti-fade with DAPI (Thermo Fisher Scientific). Slides were dried overnight and imaged by fluorescent microscope. Images were acquired at $\times 20$ and $\times 40$.

\section{FACS analysis}

Single-cell suspensions were prepared from fresh cell culture. Cell fixation and permeabilization were performed with the BD Biosciences Cytofix/Cytoperm kit (BD Biosciences), according to the manufacturer's instructions. Apoptosis and bromodeoxyuridine (BrdU) incorporation for proliferation was done using Apoptosis and Cell Proliferation Kit following the manufacturer's instructions (BD Biosciences). CD133 + population was analyzed by FACS (Miltenyi Biotech, human and mice). All samples were analyzed on BD FACSCANTO II flow cytometers (BD Biosciences). Data were acquired and analyzed with FACS DiVa software (BD Biosciences) and FlowJo Software.

\section{Sirius red staining and measurements}

Tissue sections were stained using picrosirius red staining solution (Chondrex Inc) according to the manufacturer's instructions. The Sirius red-stained area was quantified using ImageJ software by selecting stained fibers in five fields at a magnification of $\times 100$ under a light microscope. 


\section{Western blotting}

To analyzed protein expression, cells were lysed using RIPA lysis buffer (Boston Bioproducts) containing protease and phosphatase inhibitors (Roche), and protein concentration was estimated using the BCA protein estimation assay (Thermo Scientific). Equal amounts of protein were separated by SDS-PAGE and transferred to the nitrocellulose membrane. Blots were probed with antibodies against STAT3 and phospho-STAT3 (Tyr705) (Cell Signaling), after washing re-probed with secondary antibody (Abcam). The bands were visualized using super signal West Dura Extended Duration Subject (34075Thermo Fisher) in a ChemiDoc (Bio-Rad).

\section{Activity assay and lactate measurement}

Mia-PaCa2 and SU.86.86 cells grown in $70 \%$ confluent were treated with IL6 in the presence or absence of prior anti-IL6-R antibody or static treatment for $24 \mathrm{~h}$. Untreated cells were served as control. Proteins were extracted, and an equal amount of protein was used for various enzyme activity assay. ALDH, mitochondria complex 4, and PDH enzyme activity assay were performed using the respective assay kits (Abcam) according to the manufacturer's instruction. Total lactate from the cell lysates was measure using Lactate Assay Kit (Sigma, MAK064) according to the manufacturer's instruction.

\section{Extracellular acidification rate (ECAR) determination using Seahorse}

MiaPaCa-2 cells were plated at a density of 20,000 cells in a Seahorse XF 96-well plates per well in $80 \mu \mathrm{L}$ of DMEM media (with 10\% FBS) and the plate was kept $1 \mathrm{~h}$ at room temperature followed by incubation at $37^{\circ} \mathrm{C}$ with $5 \% \mathrm{CO}_{2}$ for $3 \mathrm{~h}$. Finally, $100 \mu \mathrm{L}$ of fresh media was added to have a total $180 \mu \mathrm{L}$ per well and incubated for $16 \mathrm{~h}$. Later on, cells were treated with IL6 with or without antiIL6-R antibody, the culture medium was replaced with glucose-free XF24 Seahorse medium containing $1 \mathrm{mM} 1-$ glutamine. Glycolytic flux (basal glycolysis, glycolytic capacity, and glycolytic reserve) assessed by extracellular acidification rate (ECAR) was measured by the sequential addition of glucose $(10 \mathrm{mM})$, oligomycin $(1 \mu \mathrm{M})$, and 2 DG $(50 \mathrm{mM})$ in an XF 96 Extracellular Flux Analyzer according to the manual of XF glycolysis stress test kit (no. 103020-100, Seahorse Bioscience). The ECAR values were normalized to the cell counts in each well. Glycolytic capacity was calculated according to company instruction.

\section{Glucose uptake assay}

A cell-based glucose uptake assay kit (Cayman Chemicals) was used for measuring glucose uptake in MIAPaCa2 and SU86.86 cells. Cells were plated and treated with IL6/PSC-CM/TNF $\alpha$ in the presence or absence of $30 \mathrm{~min}$ prior to anti-IL6-R antibody/static or STF31 treatment.
For labeling, isolated cells were starved in glucose-free media for $30 \mathrm{~min}$ prior to labeling with $150 \mu \mathrm{g} / \mathrm{ml} \mathrm{2-}$ NBDG, a fluorescent analog of deoxyglucose. Following incubation for $1 \mathrm{~h}$, the labeled cells were analyzed by flow cytometry (BD Biosciences) or microplate reader (Spectramax-ix3) according to the manufacturer's instruction.

\section{Animal experiment \\ Tumor initiation assay}

Male athymic nude mice between the ages of 4 and 6 weeks were purchased from the Jackson Laboratory, Bar Harbour, ME, USA. For the tumor initiation experiments, the subcutaneous mice tumor model was exploited since the appearance of tumors needed to be documented for tumor initiation. CD133- MIAPaCa-2 cells were implanted with human PSC cells in a ratio of (1:9) in the right flank of mice. Cells were implanted at a limiting dilution manner such as (100:900, 1000:9000, and 10,000:90,000). $N=5-8$ mice were used in each group. Corning $^{\circledR}$ Matrigel $^{\circledR}$ Growth Factor Reduced (GFR) Basement Membrane Matrix, purchased from Corning, Inc, Corning NY, USA, and $1 \times$ PBS at a ratio of 1:1 were used as a suspension medium for the cells. The appearance of detectable tumor nodule was observed three times in a week. The days of tumor appearance were noted in each group. Tumor initiation frequency was calculated using ELDA assay.

\section{Enrichment of CD133 + cells in vivo}

To see the effect of PSC in TIC plasticity, CD133MIAPaCa-2 cells were implanted with human PSC cells in a ration of (1:9) in male athymic nude mice. Tumor progression was monitor by measuring the tumor volume weekly. All mice were sacrificed at the end of the experiment, and the tumor was subjected to flow cytometry analysis for CD133 enrichment.

\section{Anti-IL6R treatment in an orthotopic model}

C57Bl6 mice were purchased from the Jackson Laboratory, Bar Harbour, ME, USA. In all, 2000 KPC tumor cells (KPC001) with 18,000 CAF cells were suspended in Matrigel (BD Biosciences) and injected orthotopically in the pancreas of $16 \mathrm{C} 57 \mathrm{BL} / 6$ mice. After 10 days, mice were randomly divided into two groups: Group 1 received $500 \mu \mathrm{g}$ of anti-IL-6RAb (Bio-X-cell) in every third day for 4 weeks. While mice received isotype control Ab serve as a control. All the mice were sacrificed and tumor volume and weight were measured.

\section{STAT3 inhibition in vivo}

In vivo, STAT3 inhibition was assessed using Ptf1a ${ }^{\text {Cre }}$, LSL-Kras ${ }^{G 12 D /+}$, Tgfbr $2^{\text {flox/flox }}$ (PKT) transgenic mice, which rapidly develop pancreatic tumors at 4 weeks of age $^{32}$. In brief, PKT mice were treated with vehicle or the 
STAT3 inhibitor ruxolitinib $(12 \mathrm{mg} / \mathrm{kg} /$ day, three times weekly) by oral gavage for 2 weeks prior to sacrifice and histologic tissues obtained for further analysis.

\section{Inhibition of carbonic anhydrase with WBI-5111 (aka SLC- $0111)^{33}$}

For this, KPC and CAF cells were implanted orthotopically in the pancreas of C57BL6 mice in a ratio of 1:9. Treatment with $100 \mathrm{mg} / \mathrm{kg}$ CA9 inhibitor, WBI-5111 (a gift from Welichem Biotech Inc.) was started 7 days following implantation. Three injections of $100 \mu \mathrm{g}$ anti-PD1 antibody were administered on days 17,18 , and 21 . Animals were sacrificed 30 days after the start of treatment with CA9 inhibitors.

\section{Statistical analysis}

Data were presented as means \pm SEM. Statistical analyses were performed using GraphPad Prism, version 8.0. Differences between two groups were analyzed by Student's $t$ test. $P<0.05$ was considered statistically significant. The experiments were repeated at least in triplicate three times.

\section{Ethics statement}

All animal studies were performed according to the protocols approved by IACUC at the University of Miami, USA, in accordance with the principles of the Declaration of Helsinki. All authors had access to all data and have reviewed and approved the final paper.

\section{Results}

\section{Stroma promotes tumor "initiation" and enrichment of self-renewal gene expression}

Tumor microenvironmental niches have been implicated in enriching for "cancer stem cells" or "tumorinitiating cells" in a number of cancers. Previously published studies have revealed that hypoxia enriches for pancreatic tumor-initiating cells that are aggressive and highly metastatic ${ }^{7}$. To study if the stromal fibroblasts affected an enrichment of CD133 + population, we treated human pancreatic cancer cells MIA-PaCa2 and SU86.86 with conditioned media from human PSC. Our results showed a distinct enrichment of $\mathrm{CD} 133+$ population in both cell lines (Fig. 1A, B). Treatment with PSC conditioned media also increased the invasiveness of $\mathrm{CD} 133^{\text {lo }}$ cells in vitro (as seen by Boyden chamber assay) (Fig. 1C) as well as increased expression of genes involved in cancer metastasis (Fig. 1D). Additionally, treatment with PSC conditioned media also upregulated prosurvival genes like HSP70, HSF1, NF-kB, and Survivin (Fig. 1E), indicating enrichment of aggressive, metastatic cells with an enhanced survival advantage. To further see if stroma promoted tumorigenesis in vivo, we implanted CD133- MIA-PaCa2 cells with and without PSC in a 1:9 ratio. Our results showed that while all animals in the set that was implanted with the PSCs had tumor initiation by day 37, the set of animals lacking PSC $3 / 5$ animals failed to form tumors by the end of the study at day 70 (Fig. 1F). Furthermore, we observed an increased CD133 + population in the tumors implanted with the PSCs compared the those that lacked PSC (Fig. 1G). Calculation of tumor initiation frequency by ELDA ${ }^{34}$ showed an almost 6-fold increase in the presence of stroma (Fig. 1H). Additionally, tumors lacking PSC showed a delayed tumor growth compared to the ones that had PSC co-implanted with them (Fig. 1I).

\section{Enrichment of CD133 + aggressive population in the presence of stroma is mediated by stromal IL6}

Since treatment with PSC conditioned media enriched for an aggressive population and co-implanting CD133 $3^{\text {lo }}$ cells with PSC led to increased tumor initiation, we next analyzed the PSC conditioned medium for secreted factors. Our multiplexed analysis showed that the PSC conditioned media had a significant high IL6 and TNF $\alpha$ in it (Fig. 2A). To study if the pancreatic stroma produced these cytokines during tumor progression, we next isolated murine pancreatic stellate cells (mPSC) from the pancreas of the Kras ${ }^{\mathrm{G} 12 \mathrm{D}}$; $\mathrm{TP} 53^{\mathrm{R} 172 \mathrm{H}}$; Pdx-Cre or KPC mouse model of pancreatic cancer as a pre-cancerous stage (i.e from 1-month-old animals), from the midcancer stage (i.e., when the PSCs start getting activated) and from full tumor-bearing animals (when the PSC have completely transformed to CAFs). An analysis of the secretion of cytokines from these cells showed a similar increase in IL6 and TNF $\alpha$ as the tumor progressed (Fig. 2B). The histology of the KPC animal-derived tumors showed progressive development of stroma (observed by $\alpha$ SMA staining) at these stages (Fig. $2 \mathrm{C}$ ). Since the predominant cytokine produced by the stromal fibroblasts was IL6 and TNFo, we next treated the MIA-PACA2 and SU86.86 cells with IL6 and TNF $\alpha$. While treatment with IL6 showed a significant increase in the CD133 population (Fig. 2D, E), treatment with TNF $\alpha$ did not show any significant enrichment (Supplementary Fig. 1A). We thus focused our effort on IL6. To test the specificity of IL6 in enriching for CD133+ population, we treated the cells with an anti-IL6R antibody for $1 \mathrm{~h}$ before treating with IL6. Our results showed that IL6 mediated increase in CD133+ population was decreased in the presence of anti-IL6R $A b$, indicating that this was enrichment was being orchestrated by IL6. Similarly, when these cells were pretreated with anti-IL6 Ab and then exposed to PSC conditioned media, there was a significant decrease in the CD133+ population (Fig. 2D, E). Additionally, IL6 mediated increase in expression of self-renewal genes was decreased when cells were pretreated with anti-IL6R Ab (Fig. 2F). Aldehyde dehydrogenase activity is associated 


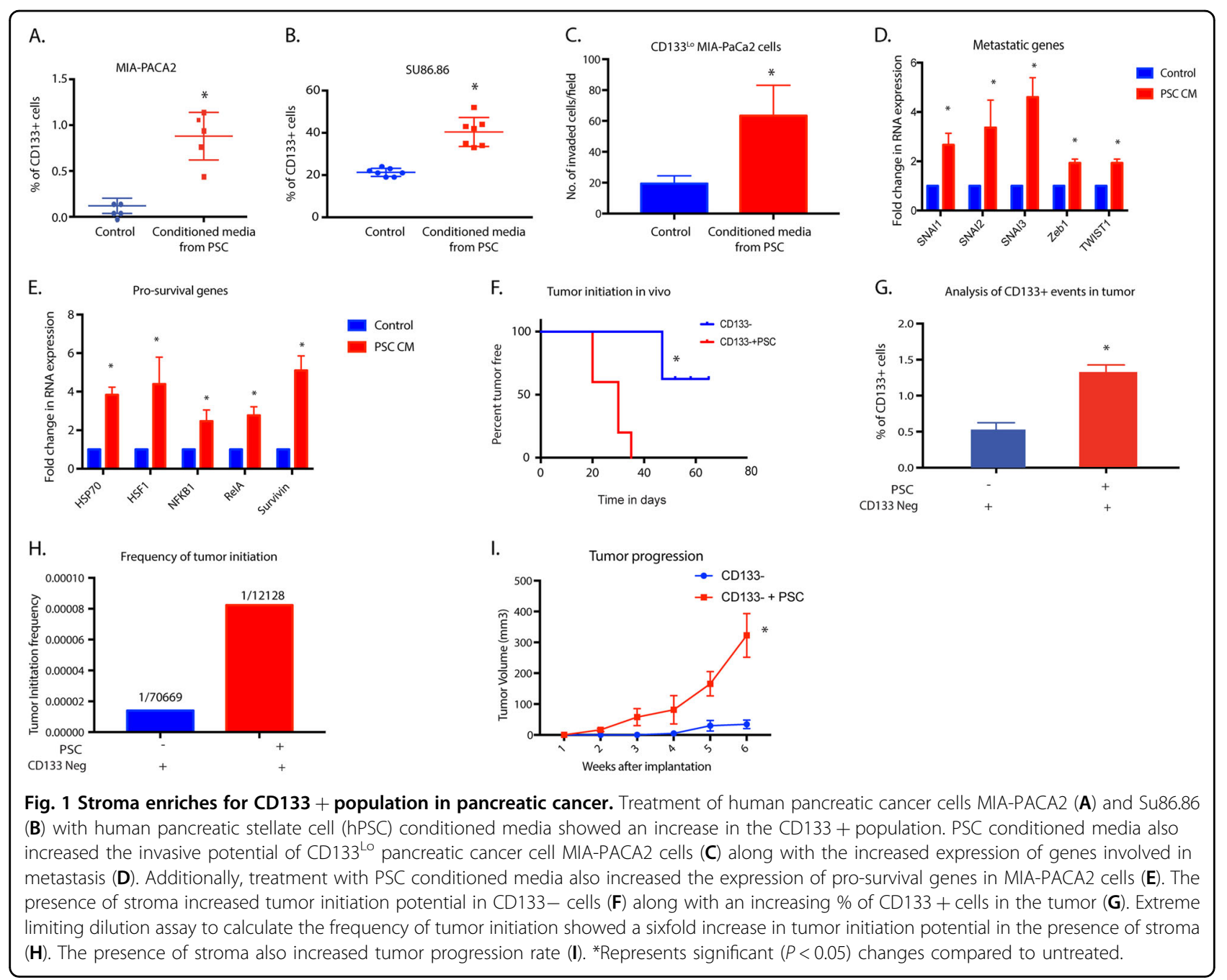

with an aggressive and "stem-like" population represented that are CD133 $+{ }^{35}$. Our results showed an increase in Aldh activity that was decreased upon treatment with anti-IL6R Ab (Fig. 2G). Consistent with this, pretreatment of pancreatic cancer cells with IL6 also increased their resistance to gemcitabine and reduced apoptosis in these cells (Supplementary Fig. 2).

\section{Stromal IL6 increases glucose uptake and flux through glycolysis in enriched CD133 + cells}

Previously published results from our laboratory showed that CD133 + cells had increased metabolic flux through the glycolytic pathway and a very rudimentary oxidative phosphorylation ${ }^{8}$. This contributed to the upregulation of anti-apoptotic and pro-survival genes in this population. To determine if the IL6 secreted by CAFs altered the metabolic profile of the cells as they enriched for CD133 + cells, we performed an energy phenotyping assay on SU86.86 and MIA-PACA2 cells using a Seahorse platform. Our results showed that upon treatment with
IL6, both cell lines had increased extracellular acidification rate or ECAR (Fig. 3A, B). Further, glycolytic capacity (Fig. 3C) was increased with IL6 in both cell lines and decreased when signaling was blocked by with anti-IL6R antibody. We next confirmed this by estimating the lactate secreted by the cells. Our results showed that increased lactate was produced by tumor cells in the presence of IL6. This was decreased when IL6 was inhibited using an anti-IL6R antibody (Fig. 3D). We next determined if the gene expression of the glucose utilization enzymes was affected by stromal IL6 by using a PCR array. Our results showed that treatment with stromal IL6 increased genes involved in glucose metabolism, specifically glycolysis, that decreased when IL6 receptor on tumor cells was silenced by siRNA for IL6R in SU86.86 (Fig. 3E, F). The silencing of IL6R was confirmed by qRTPCR (Supplementary Fig. 1B). To determine if increased glycolysis and thus ECAR in the presence of stromal IL6 is mediated by increased glucose transporter activity, we measured glucose uptake (using fluorescent 


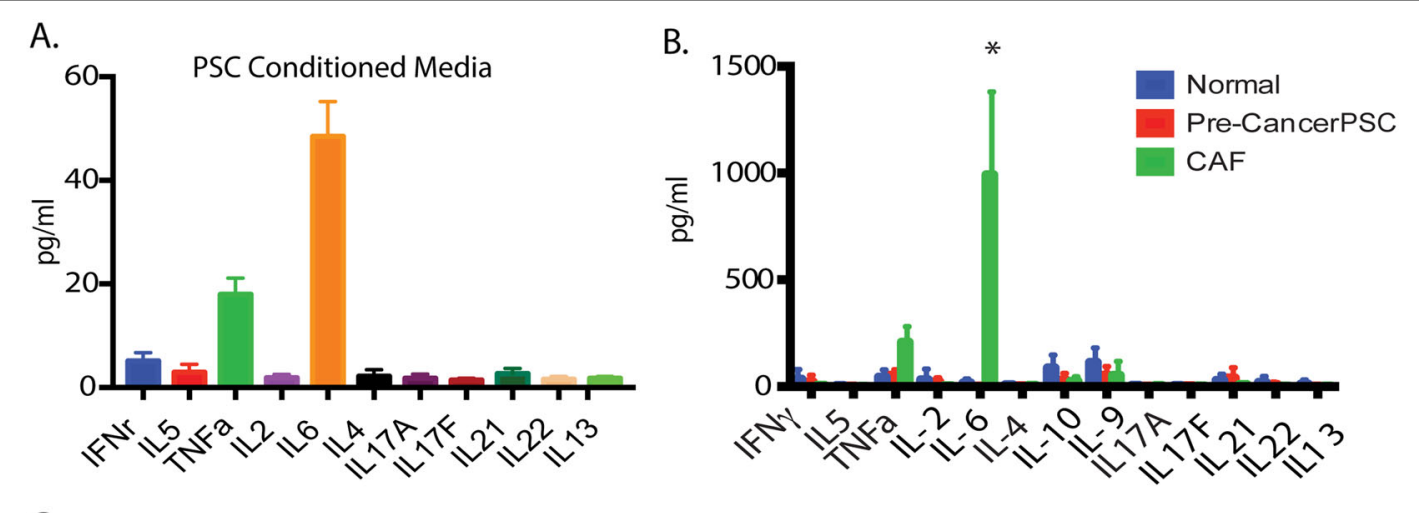

C.

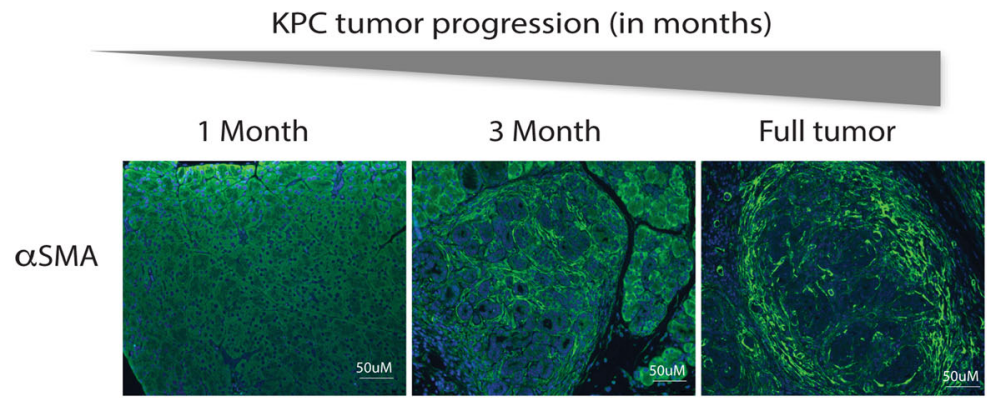

D.

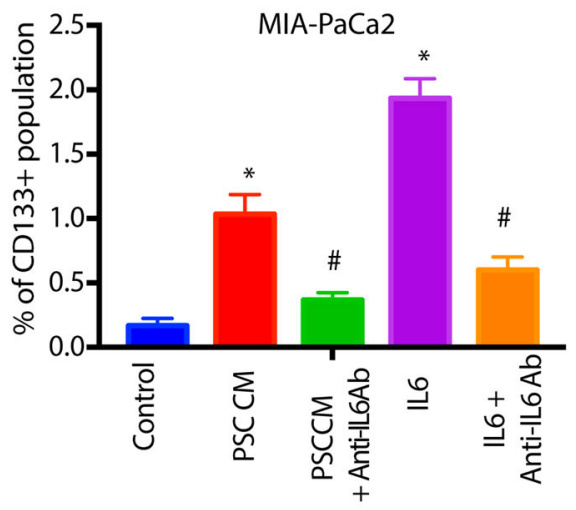

F.

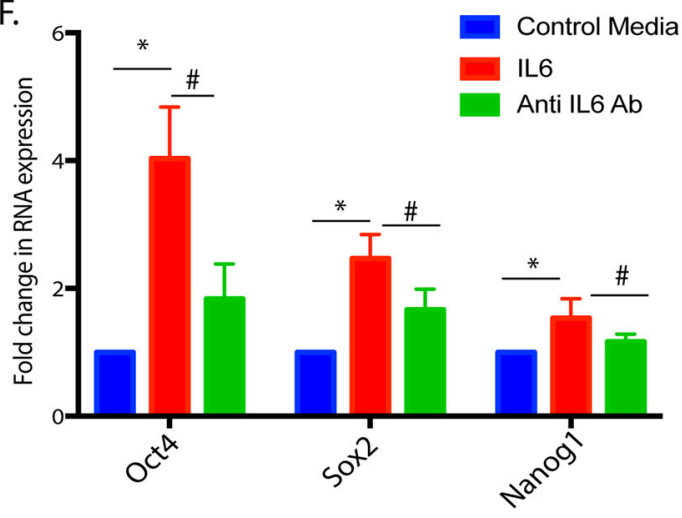

E.

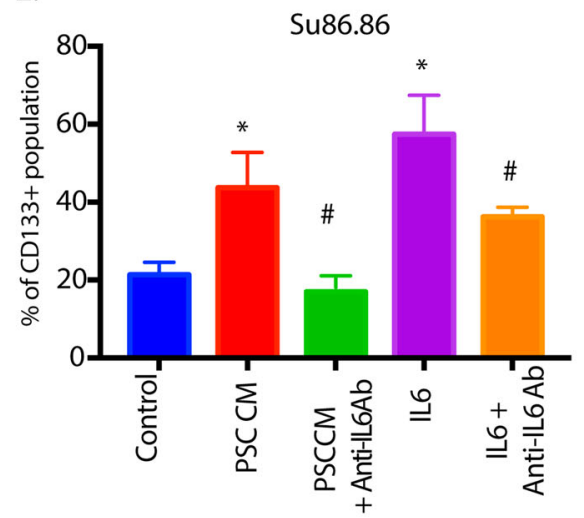

G.

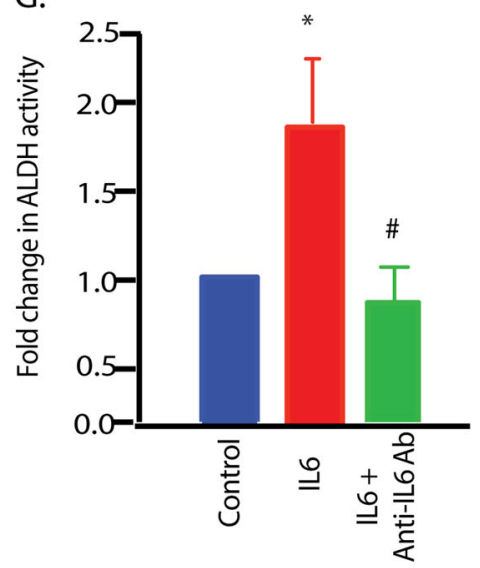

Fig. 2 (See legend on next page.) 
(see figure on previous page)

Fig. 2 IL6, the primary cytokine produced by stromal cells enriches for CD133 + population. Analysis of PSC conditioned media showed IL6 to be the main cytokine produced by the PSC cells in culture (A). Similar secretion of IL6 was observed when normal, precancer PSC, and CAFs from full tumors were analyzed for secreted cytokines (B). Pancreatic cancer stroma develops as the tumor progresses as observed by the increased expression of alpha SMA in tumors (C). An increase in CD133 + cells upon IL6 treatment or PSC treatment was observed to be reduced with IL6 signaling was blocked using the anti-IL6R antibody in both MIA-PACA2 (D) and SU86.86 cells (E). Treatment with IL6 increased stemness genes (F) and ALDH activity $(\mathbf{G})$, which were reversed when signaling was blocked with the anti-IL6R antibody. ${ }^{*}$ Represents significant $(P<0.05)$ changes compared to untreated and \# represents significant $(P<0.05)$ changes compared to IL6 treatment.
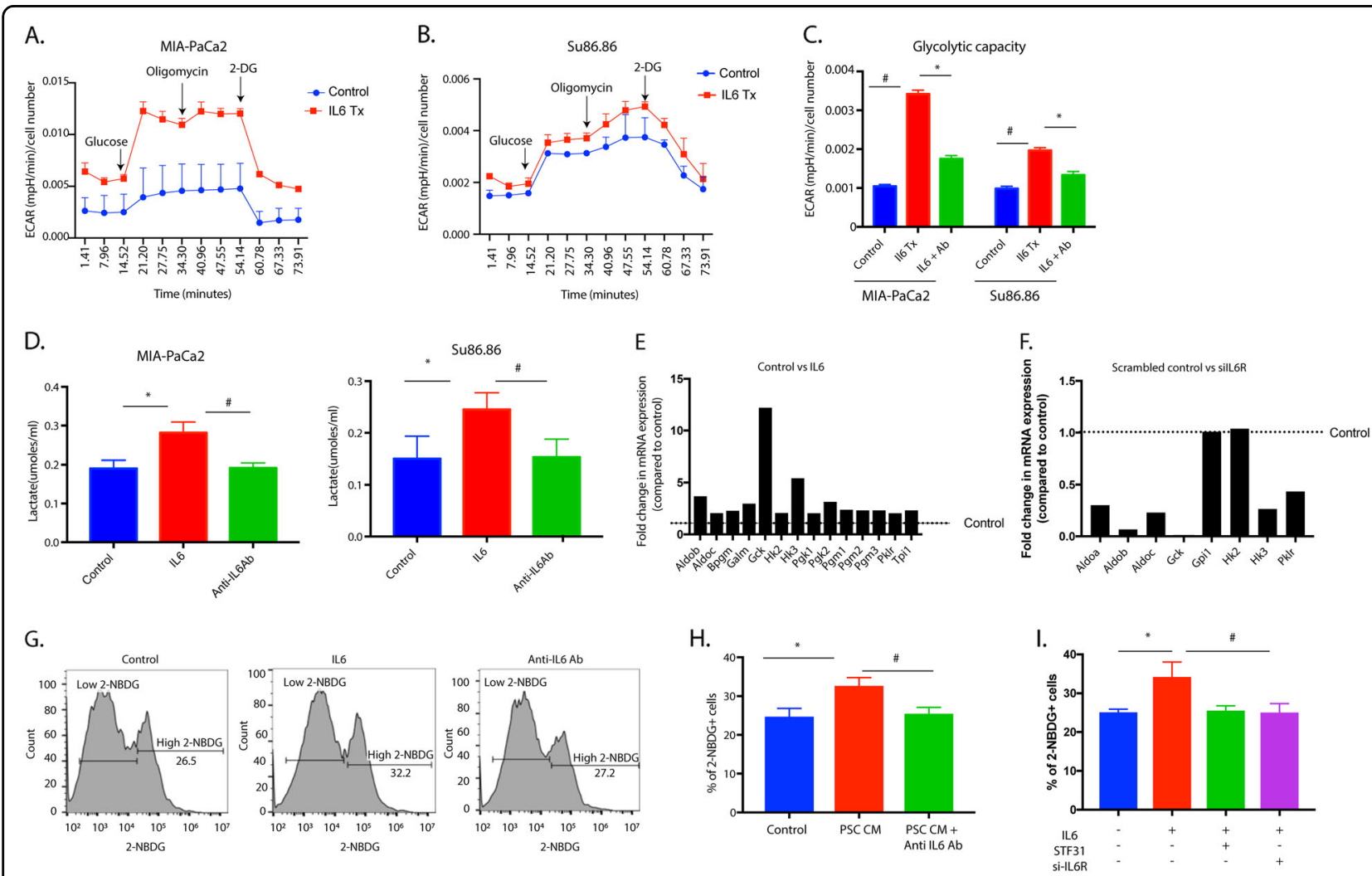

Fig. 3 Treatment with IL6 alters metabolic profile in pancreatic cancer cells. Treatment with IL6 increased ECAR in MIA-PACA2 (A) and SU86.86 cells (B) and increased the glycolytic capacity of the cells (C). An increase in lactate production was also observed in both MIA-PACA2 and SU86.86 cells (D), which was reduced when IL6 signaling was blocked. The expression of genes involved in glucose metabolism was altered upon IL6 treatment (E). Similarly, blocking IL6 signaling by silencing the IL6 receptor showed a downregulation of glucose metabolism genes (F). IL6 also increased glucose uptake as observed by 2-NBDG uptake assay by flow cytometry (G). Glucose uptake was similarly increased with SU86.86 cells were treated with PSC conditioned media $(\mathbf{H})$. This effect was reverted upon blocking IL6 signaling. Additionally, inhibition of glucose transporter using 5 UM STF31 or by silencing IL6R decreased the $\%$ of 2-NBDG + cells. *Represents significant $(P<0.05)$ changes compared to untreated and \# represents significant $(P<0.05)$ changes compared to IL6 treatment.

glucose analog 2-NBDG) by the tumor cells after blocking IL6 receptor (using anti IL6R antibody) and glucose transporter inhibitor STF31. Our results showed increased glucose uptake in the presence of both PSC-CM and IL6 treatment in Su86.86 cells, which was abrogated by treatment with anti-IL6R antibody (Fig. 3 G, H). Similar results were observed in MIA-PaCa2 cells as well (Supplementary Fig. 1C). We further observed that the effect of IL6 was reverted upon silencing the IL6 receptor on tumor cells (by siRNA for IL6R) as well as upon inhibition of glucose transporters by STF31 (Fig. 3I). This indicated that stromal IL6 mediated increased glycolysis and ECAR in pancreatic tumor cells by increased glucose uptake via glucose transporters.

\section{Stromal IL6 induces metabolic reprogramming and enrichment of CD133 + population via STAT3 signaling}

To study if stromal IL6 was activating the canonical JAK/ STAT pathway in the tumor cells, we next evaluated the activation of STAT3 by treating with IL6. Our studies 

A.
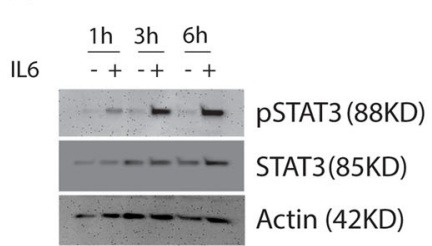
Su 86.86 Actin (42KD)
B.

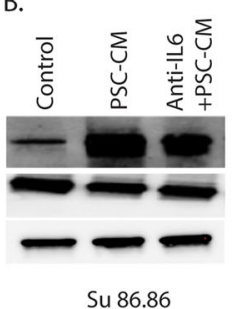
pSTAT3 (88KD)
STAT3 (85KD)
Actin (42KD)
Su 86.86
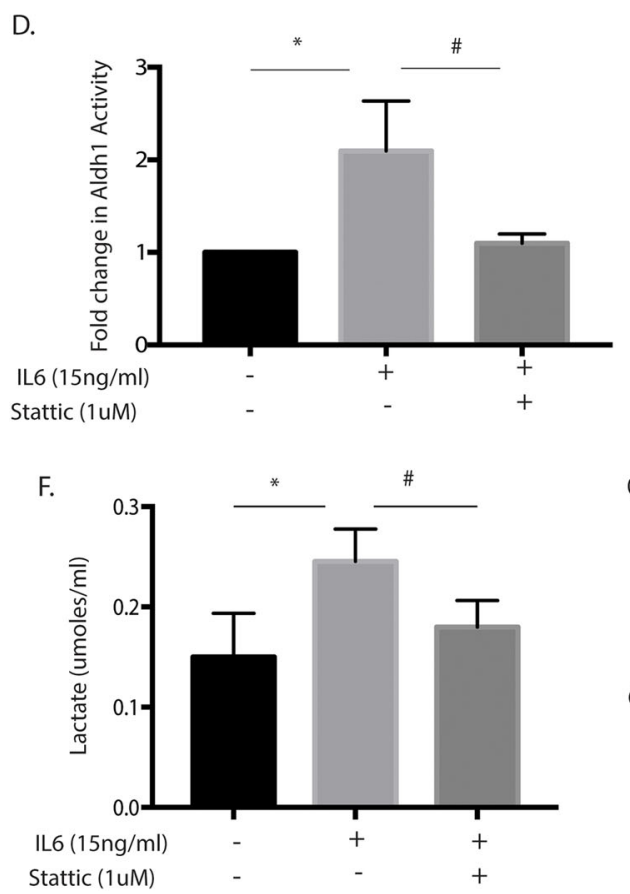

G
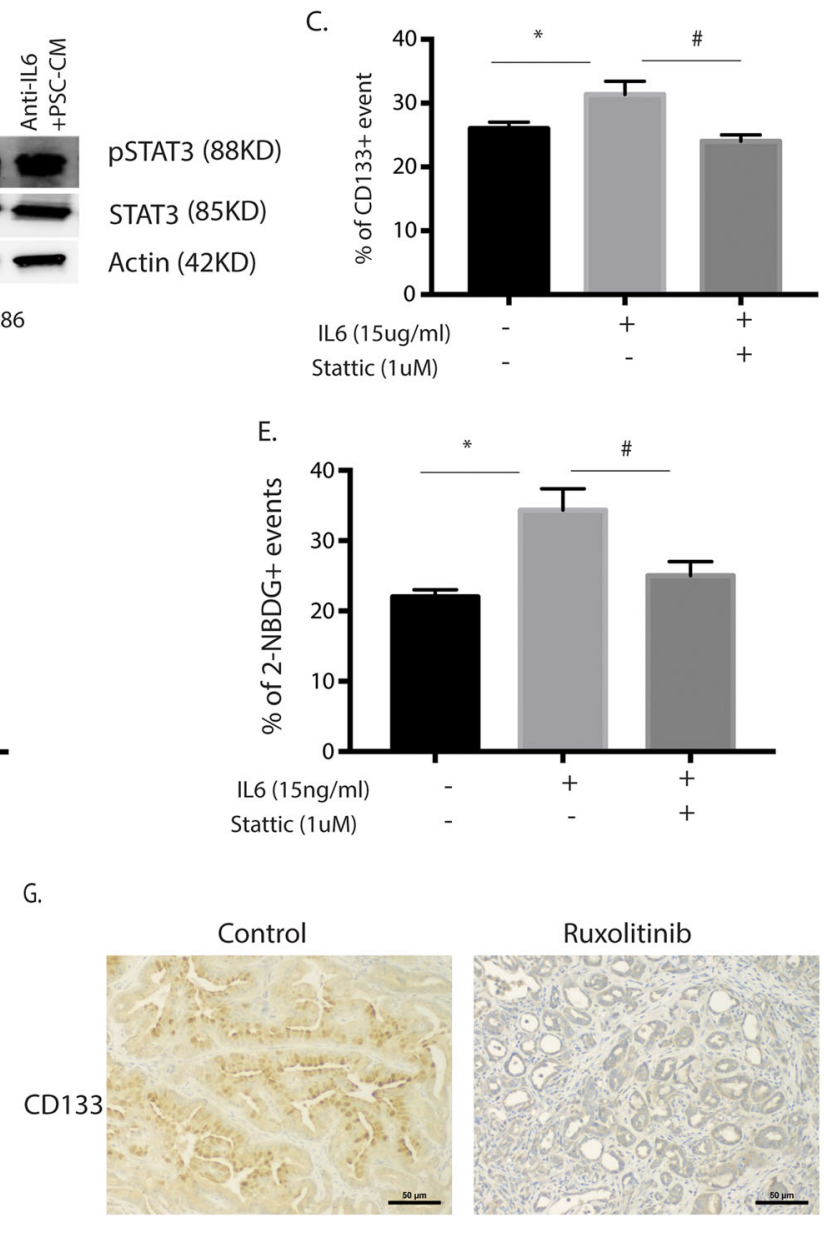

Fig. 4 IL6 induced an increase in stemness and metabolic changes are mediated via STAT3 signaling. Treatment with IL6 (A) or PSC conditioned media (PSC-CM) induced activation of STAT3 in SU86.86 cells (B). IL6 induced an increase in CD133 + population (C) ALdh1 activity (D), glucose uptake $(\mathbf{E})$, and extracellular lactate $(\mathbf{F})$ was reduced when STAT3 signaling was blocked by small-molecule inhibitor Stattic. Treatment of pancreatic tumors in PKT mice with ruxolitinib, a STAT signaling inhibitor also decreased CD133 + cells in the tumor tissues as seen in the representative pictures $(\mathbf{G})$. ${ }^{*}$ Represents significant $(P<0.05)$ changes compared to untreated and \# represents significant $(P<0.05)$ changes compared to IL6 treatment.

showed STAT3 was activated (as seen by its phosphorylation as early as $1 \mathrm{~h}$ and remained activated for $6 \mathrm{~h}$ (Fig. $4 \mathrm{~A}$ and Supplementary Fig. 3A, B). Similar phosphorylation of STAT3 was observed with PSC conditioned media as well (Fig. 4B and Supplementary Fig. 3C). We next evaluated if the enrichment of $\mathrm{CD} 133$ + population, as well as the metabolic changes observed in pancreatic cancer cells, were dependent on activation of STAT3. To determine this, we stimulated pancreatic cancer cells with IL6 in the presence of a STAT3 inhibitor Stattic. Our results showed that inhibition of STAT3 signaling in the presence of IL6 decreased CD133 + population (Fig. 4C and Supplementary Fig. 3D), and Aldh activity (Fig. 4D). Additionally, treatment with $1 \mu \mathrm{M}$ Stattic in the presence of IL6 decreased glucose uptake (Fig. 4E and Supplementary Fig. 3B) as well as lactate production (Fig. 4F and Supplementary Fig. 3E) in pancreatic cancer cells Su86.86 and MIA-PACA2. In vivo, treatment with ruxolitinib, an inhibitor of STAT signaling pathway also reduced $\mathrm{CD} 133$ + cells in pancreatic cancer tissues from PKT mice (Fig. 4G).

\section{Stromal IL6 prevents glucose from entering oxidative phosphorylation}

Our gene expression analysis following IL6 treatment and an IPA analysis revealed that the increased glycolysis was also associated with a decreased transcription of some of the mitochondrial activity genes like PDHA, KGDH, aconitase, and SDHA-D (Fig. 5A). One of the proteins that regulate the entry of glycolytic intermediates to the mitochondria and oxidative phosphorylation is PDHA. To 


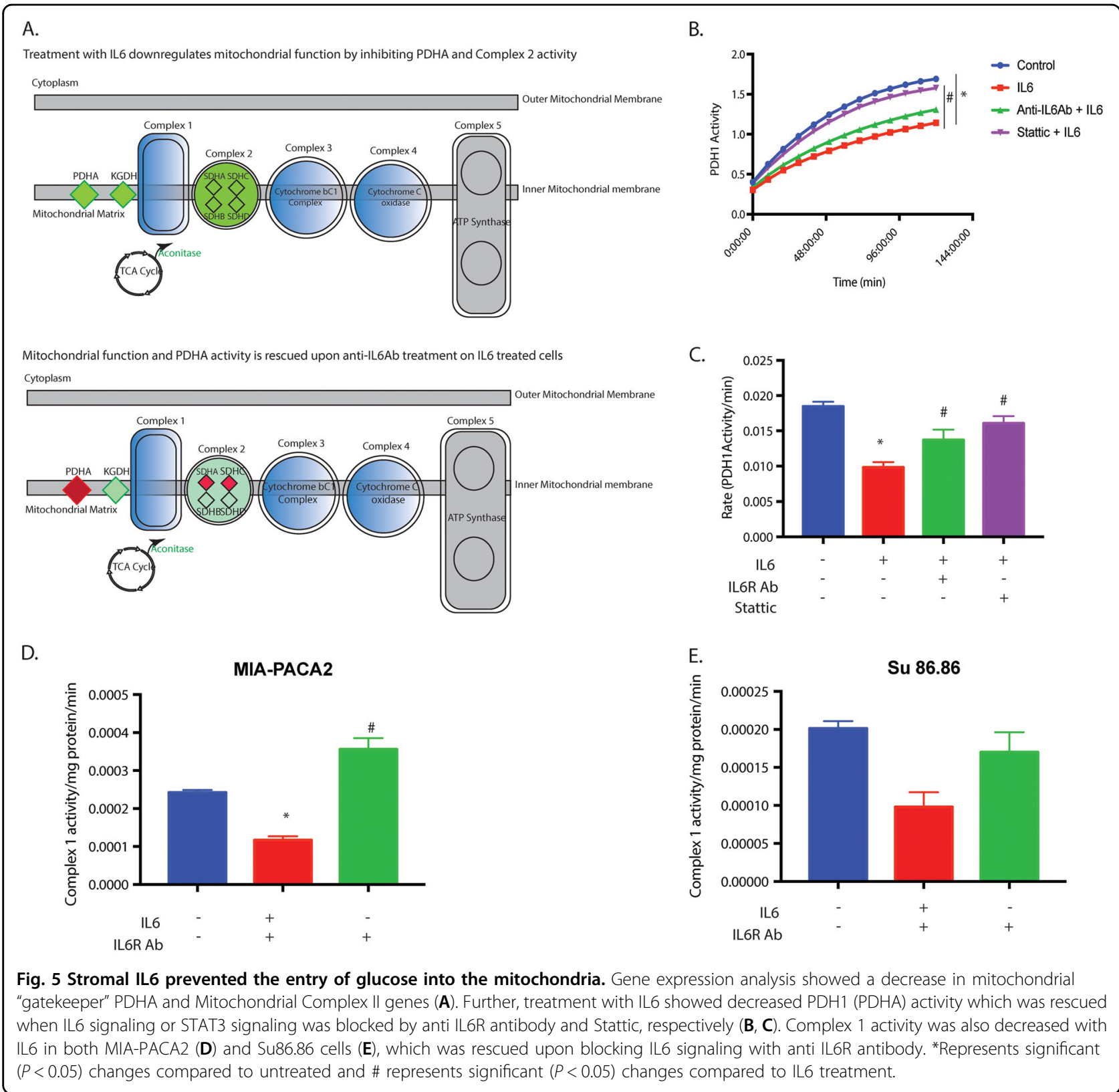

determine if treatment with IL6 decreased the activity of PDHA, we estimated the activity of PDH1 SU86.86 cells. Our result showed that while treatment with IL6 decreased PDH1 activity, pretreatment with anti-IL6R antibody or Stattic reverted this inhibition (Fig. 5B, C). In MIA-PaCa2 cells; however, this effect was not as pronounced as in Su86.86 cells (Supplementary Fig. 3F), IL6 decreased PDHA activity, however, pretreatment with anti-IL6R and/or stattic showed a modest recovery. In addition, IL6 treatment inhibited mitochondrial complex 1 activity, which was recovered once signaling was blocked by IL6R antibody in both MIA-PaCa2 and Su.86.86 cells (Fig. 5D, E). This indicated that stromal IL6 increased glycolysis in cancer cells and suppressed the entry of glucose to oxidative phosphorylation. Interestingly, treatment with IL6 did not affect the OCR in these cells significantly (data not shown).

\section{Inhibition of IL6 inhibits pancreatic tumor progression}

IL6 has been shown to be involved in the progression of pancreatic tumors by others ${ }^{16,36-39}$. Treatment of pancreatic cancer cells with IL6 or conditioned media from PSC confirmed this in our in vitro experiment (Supplementary Fig. 4A, B). Furthermore, in the pancreatic cancer patient population, increased expression of IL6 correlated with poor survival (Fig. 6A). To confirm this in 


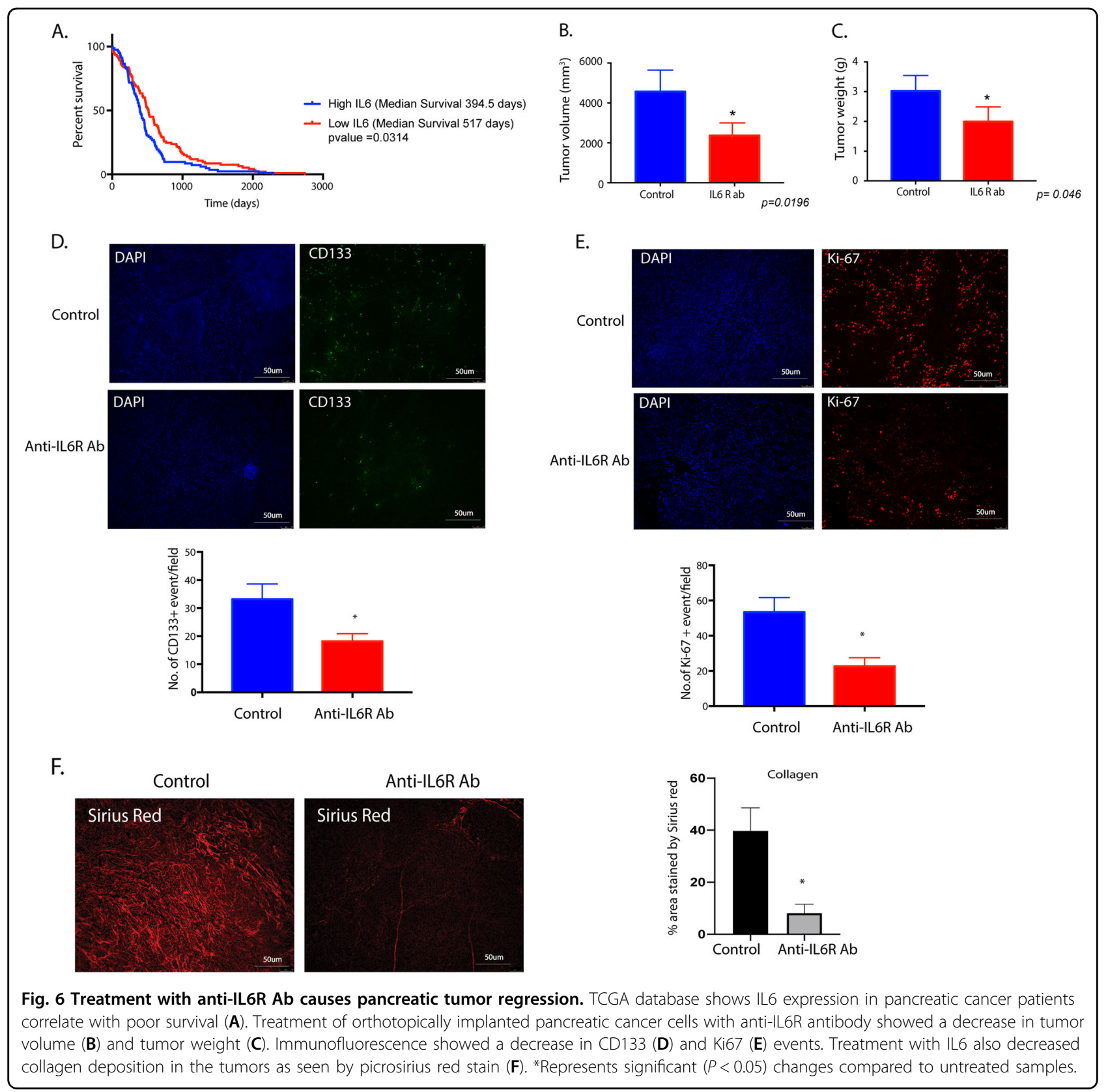

our animal model, we next implanted KPC001 cells with CAF cells orthotopically in the pancreas of the C57Bl6 mice in a ratio of 1:9. Seven days following implantation, the animals were treated with anti-IL6R Ab. After 30 days of treatment, our results showed a significant decrease in tumor volume (Fig. 6B) and tumor weight (Fig. 6C). Further, the analysis of the histology of these slides further showed a decrease in CD133 + population in the antiIL6R antibody-treated group (Fig. 6D). In addition, H\&E and Ki67 staining showed a general decrease in proliferative cells (Fig. 6E). We next evaluated the collagen deposition in the pancreatic tumor microenvironment.
Treatment with anti-IL6R antibody showed significant depletion of collagen in the tumors (Fig. 7A) compared to the control animals (Fig. 6F).

Inhibition of the IL6, as well as effluxed lactate by CA9 inhibitor, modulated pancreatic tumor microenvironment

To determine the effect of IL6 inhibition on macrophage polarization, we next stained the anti-IL6 Abtreated tumors with pan macrophage marker F4/80 and M2 marker CD206. A ratio of CD206:F4/80 revealed that anti-IL6-treated tumors had decreased M2 macrophages compared to the control animals (Fig. 7A, B). Along with 


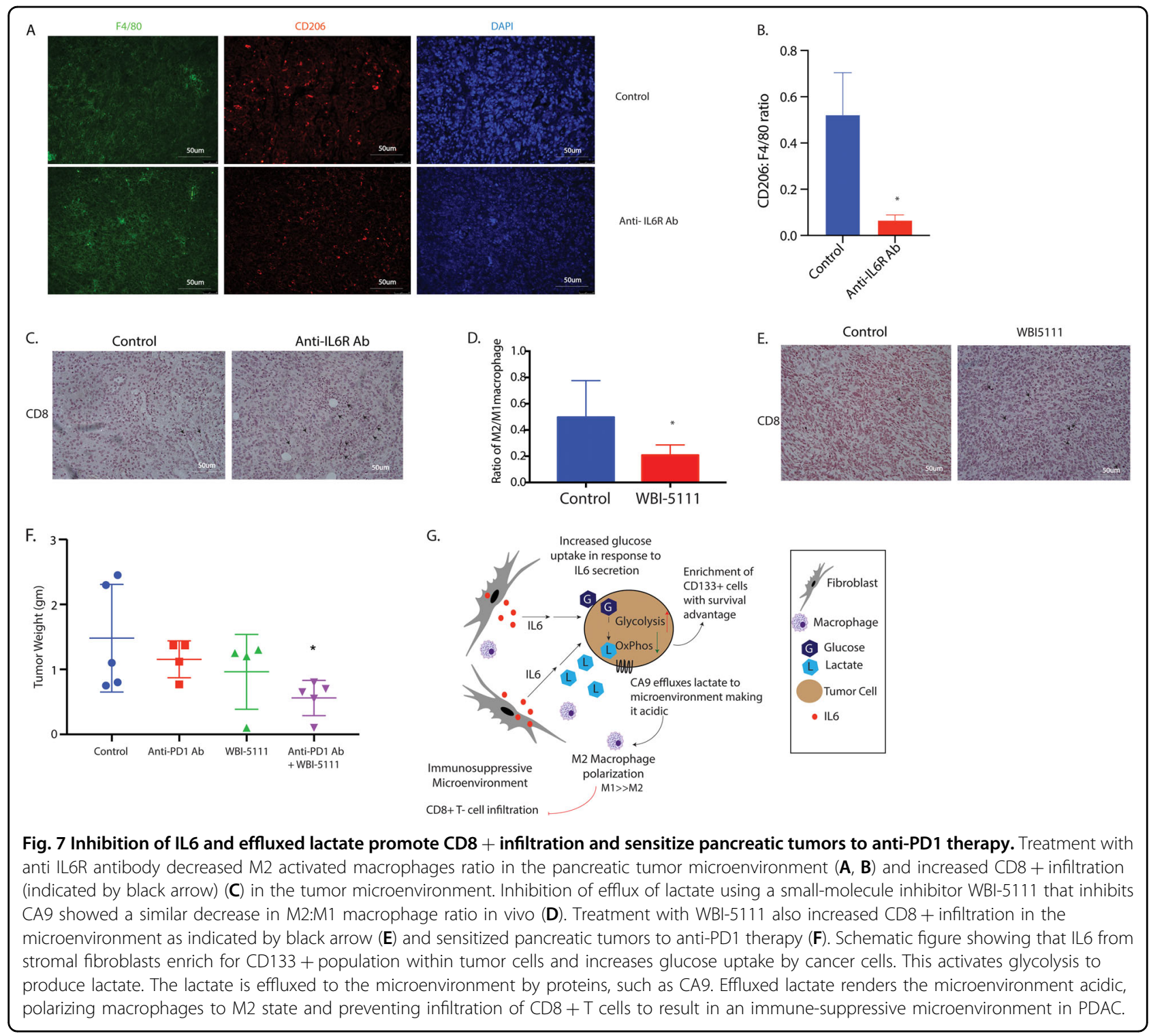

these, there was an increased infiltration of CD8 + T cells in the tumors when IL6 signaling was blocked (Fig. 7C, and Supplementary Fig. 4C).

To further study if it was the effluxed lactate in the pancreatic tumor that contributed to the increased CD8 + infiltration, we next implanted KPC:CAF cells in the pancreas of C57BL6 animals and treated them with a CA9 inhibitor. CA9 promotes efflux of lactate from cells to prevent a lactate build up. Analysis of tumors by flow cytometry showed an increased M1/M2 ratio of macrophages within the tumor (Fig. 7D). Additionally, the analysis of $\mathrm{CD} 8+$ population within the tumors indicated an increase in the CD8 $+\mathrm{T}$ cells (Fig. 7E and Supplementary Fig. 4D). IL6 inhibition has been shown to sensitize pancreatic tumors to immune checkpoint inhibitor therapy ${ }^{40}$. To study if inhibition of lactate efflux affected the response of pancreatic tumors to anti-PD1 therapy, we next evaluated the effect of CA9 inhibitor in combination with anti-PD1 therapy. Our results showed that this combination was significantly better in decreasing pancreatic tumors than either therapy alone (Fig. 6F).

\section{Discussion}

Many studies have defined the "stem-like" CD133+ population as the "Tumor Initiating Cell or TIC population. However, how this population arises in a tumor has remained enigmatic. Our studies show that CD133+ population in a pancreatic tumor can form tumors at very low dilutions ${ }^{5}$. Further, overexpression of CD133 in a cell line that lacks the expression of this gene (MIA-PACA2) results in the induction of self-renewal properties in these cells ${ }^{6}$. This establishes that CD133 + population is an 
accurate representation of the aggressive treatmentrefractory population in pancreatic cancer. By definition, TICs have the ability to form tumors that recapitulate the heterogeneity of the primary tumor from which they were isolated following orthotopic transplantation into mice $^{41,42}$. As the progeny of TICs differentiate, they lose their ability to initiate tumors, despite their identical genetic landscape and become "non-TIC" population. However, studies indicate these CD133 + populations are not necessarily the "cell of origin" for pancreatic cancer and thus tumor-initiating cell per se but rather a population that is responsible for recurrence, owing to an increased survival advantage. This population of tumor cells has increased adaptation to unfavorable microenvironments like hypoxia and nutrient deprivation. These heightened adaptations to stress in this CD133+ population gives them a distinct advantage over the CD133- population in tiding through unfavorable conditions until the host environment is conducive for tumor relapse at the primary or metastatic site. Thus, in essence, these cells are the "roots" of aggressive tumors for which there is currently no effective treatments ${ }^{4}$. Our current study shows that in the presence of stroma, there is an enrichment of CD133 + population within pancreatic tumors (Fig. 1).

The role of the stromal cells in the pancreatic tumor microenvironment has gained importance in the last decade. Yet, how the stroma influences the properties of the tumor has remained unknown. A study by Ohlund et al showed the heterogeneity in the cancer-associated fibroblasts (CAFs) in pancreatic tumors, classifying them into inflammatory CAFs (iCAFs) and myofibroblastic CAFs or myCAFs ${ }^{16}$. This study also showed that inflammatory CAFs secreted IL6 as one of the major cytokines. This is consistent with our findings that during the progression of pancreatic cancer, as the stromal develops and possibly differentiates into iCAFs and myCAFs, there is increased secretion of IL6 (Fig. 2). Furthermore, our results show that the stroma secreted IL6 activates the STAT signaling pathway to enrich for "stem-like" CD133 + cells as well as alter the metabolic profile of the pancreatic cancer cells (Figs. 3 and 4). When IL6 signaling was inhibited by blocking the IL6 receptors (by either anti-IL6R antibody or by si-IL6R), a reversal of the enrichment as well as metabolic reprogramming was observed. A similar reversal was also observed when STAT3 was inhibited by inhibitor Stattic (Fig. 4). Interestingly, an analysis of STAT3 target genes (in the CHEA transcription factor target database) revealed that stemness genes like Sox2, Nanog, and Oct4 as well as certain metabolic genes like carbonic anhydrases and LDH were targets of STAT3 transcription factors. This indicated that stromal IL6, activated STAT signaling pathway to upregulate mRNA expression of both stemness genes as well as metabolic genes in pancreatic tumor cells (Supplementary Fig. 4E).

IL6 induced metabolic reprogramming in pancreatic tumor cells. We observed increased glycolysis and reduced mitochondrial activity in cells treated with IL6. Interestingly, IL6 resulted in increased production of lactate and inhibited the activity of pyruvate dehydrogenase or PDH1, a gatekeeper enzyme of mitochondrial respiration. This was further confirmed upon assaying for mitochondrial complex 1 activity. Treatment with IL6 decreased mitochondrial complex 1 activity confirming that the pancreatic tumor cells were preferentially synthesizing lactate in the presence of IL6. Lactate is effluxed in the microenvironment by the activity of CA9.

Studies have shown that this acidification of the microenvironment contributes to immune suppression by favoring polarization of macrophages to an M2 phenotype, which in turn excludes CD8 $+\mathrm{T}$ cells ${ }^{27}$. It is wellknown that pancreatic tumors have an immunesuppressive microenvironment with limited CD8+ $\mathrm{T}$ cells. However, whether this is because of effluxed lactate is not known. Our study showed that inhibiting IL6 in vivo using an IL6R antibody or by inhibiting the efflux of lactate by inhibiting CA9, decreased the M2 macrophages in the microenvironment, and increased CD8 + infiltration (Fig. 7). Remarkably, inhibiting IL6 had a striking effect on collagen in the stroma (Fig. 7A). A recently published study from our laboratory shows that inhibiting of the hexosamine biosynthesis pathway decreased collagen in the pancreatic tumors and increased CD8 + infiltration ${ }^{29}$. Whether inhibition of IL6 signaling functions similarly is unclear and beyond the scope of this paper. Increased infiltration of CD8 + cells can potentially sensitize tumors to immune therapy. Further, previous research has shown that inhibition of IL6 can sensitize pancreatic tumors to immune therapy ${ }^{40}$. To study if this overcoming of immune evasion by inhibition of IL6 signaling is due to decreased lactate efflux by tumor cells, we inhibited lactate efflux by inhibiting CA9. Our results showed that this sensitized pancreatic tumors to anti-PD1 therapy (Fig. 7). This is a significant observation in the context of pancreatic cancer since this disease is notoriously resistant to immune therapy (Fig. 7G).

Interestingly, inhibitors of JAK/STAT pathway like ruxolitinib or IL6 inhibition as monotherapy have not shown significant clinical activity in solid tumors. It is possible that the heterogeneity of the tumors is responsible for this phenomenon. However, IL6 inhibition either by neutralization or by receptor blocking has shown remarkable effects in pre-clinical models ${ }^{43,44}$.

Overall, this study shows the importance of stromal cells in promoting tumor progression by enriching for an aggressive and stem-like population within the tumor. It 
further shows that stroma can drive a metabolic reprogramming and favor glycolysis in the tumor cells to promote increased efflux of lactate in the microenvironment, that can contribute to the immune-evasive property of the tumor. Finally, our study shows that we can target the IL6-induced lactate efflux from tumor to revert the immune-evasive microenvironment to an immunesupportive one and thus augment outcomes of checkpoint inhibitor therapy.

\section{Acknowledgements}

The authors would also like to thank Oliver Umland at Diabetes Research Institute, University of Miami, and the Flow Cytometry Shared Resources at the Sylvester Comprehensive Cancer Center for their help. We would also like to thank the Shared Pathology and Histology core for their help with tissue sections and staining. We woud like to thank Dr. Shanta Dhar and her lab for help with the Seahorse experiments in the paper. This study was funded by NIH grant R01-CA184274 and R01-CA124723 (to SB) and CA161976 (to N.M.); James Esther and King Biomedical Research Program by Florida Department of Health grant 9JKO9 (to S.B.); Pancreatic Cancer Action Network grant to N.M. The research reported in this publication was supported by the National Cancer Institute of the National Institutes of Health under Award Number P30CA240139.

\section{Author details}

${ }^{1}$ Department of Surgery, Miller School of Medicine, University of Miami, Miami, FL, USA. ${ }^{2}$ Sylvester Comprehensive Cancer Center, Miami, FL, USA. ${ }^{3}$ WeliChem Biotech Inc, Vancouver, British Columbia, Canada

\section{Conflict of interest}

University of Minnesota has a patent for Minnelide, which has been licensed to Minneamrita Therapeutics, LLC. A.K.S. is the co-founder and the Chief Scientific Officer of this company. S.B. is a consultant with Minneamrita Therapeutics LLC and this relationship is managed by the University of Miami. The remaining authors declare no conflict of interest.

\section{Publisher's note}

Springer Nature remains neutral with regard to jurisdictional claims in published maps and institutional affiliations.

Supplementary Information accompanies this paper at (https://doi.org/ 10.1038/s41419-020-03168-4).

Received: 4 May 2020 Revised: 26 October 2020 Accepted: 27 October 2020 Published online: 11 November 2020

\section{References}

1. Siegel, R. L., Miller, K. D. \& Jemal, A. Cancer statistics, 2018. CA Cancer J. Clin. 68, 7-30 (2018)

2. Lee, G., Hall, R. R., 3rd \& Ahmed, A. U. Cancer stem cells: cellular plasticity, niche, and its clinical relevance. J. Stem Cell Res. Ther. 6, https:/doi.org/10.4172/21577633.1000363 (2016).

3. Maeda, M. et al. Cancer cell niche factors secreted from cancer-associated fibroblast by loss of H3K27me3. Gut 69, 243-251 (2020).

4. Adorno-Cruz, V. et al. Cancer stem cells: targeting the roots of cancer, seeds of metastasis, and sources of therapy resistance. Cancer Res. 75, 924-929 (2015).

5. Banerjee, S. et al. CD133+ tumor initiating cells in a syngenic murine model of pancreatic cancer respond to Minnelide. Clin. Cancer Res. 20, 2388-2399 (2014).

6. Nomura, A. et al. CD133 initiates tumors, induces epithelial-mesenchymal transition and increases metastasis in pancreatic cancer. Oncotarget $\mathbf{6}$ 8313-8322 (2015)
7. McGinn, O. et al. Inhibition of hypoxic response decreases stemness and reduces tumorigenic signaling due to impaired assembly of HIF1 transcription complex in pancreatic cancer. Sci. Rep. 7, 7872 (2017).

8. Nomura, A. et al. Microenvironment mediated alterations to metabolic pathways confer increased chemo-resistance in CD133+ tumor initiating cells. Oncotarget 7, 56324-56337 (2016).

9. Nomura, A. et al. Minnelide effectively eliminates CD133(+) side population in pancreatic cancer. Mol. Cancer 14, 200 (2015).

10. Crawford, H. C., Pasca di Magliano, M. \& Banerjee, S. Signaling networks that control cellular plasticity in pancreatic tumorigenesis, progression, and metastasis. Gastroenterology 156, 2073-2084 (2019).

11. Rucki, A. A. et al. Heterogeneous stromal signaling within the tumor microenvironment controls the metastasis of pancreatic cancer. Cancer Res. 77 41-52 (2017).

12. Pan, B., Liao, Q., Niu, Z., Zhou, L. \& Zhao, Y. Cancer-associated fibroblasts in pancreatic adenocarcinoma. Future Oncol. 11, 2603-2610 (2015).

13. Lo, A. et al. Tumor-promoting desmoplasia is disrupted by depleting FAPexpressing stromal cells. Cancer Res. 75, 2800-2810 (2015).

14. Aiello, N. M. et al. Metastatic progression is associated with dynamic changes in the local microenvironment. Nat. Commun. 7, 12819 (2016).

15. Bremnes, R. M. et al. The role of tumor stroma in cancer progression and prognosis: emphasis on carcinoma-associated fibroblasts and non-small cell lung cancer. J. Thorac. Oncol. 6, 209-217 (2011).

16. Ohlund, D. et al. Distinct populations of inflammatory fibroblasts and myofibroblasts in pancreatic cancer. J. Exp. Med. 214, 579-596 (2017).

17. Nagathihalli, N. S. et al. Pancreatic stellate cell secreted IL-6 stimulates STAT3 dependent invasiveness of pancreatic intraepithelial neoplasia and cancer cells. Oncotarget 7, 65982-65992 (2016).

18. Sousa, C. M. et al. Pancreatic stellate cells support tumour metabolism through autophagic alanine secretion. Nature 536, 479-483 (2016).

19. Auciello, F. R. et al. A stromal lysolipid-autotaxin signaling axis promotes pancreatic tumor progression. Cancer Discov. 9, 617-627 (2019).

20. Sanford-Crane, H., Abrego, J. \& Sherman, M. H. Fibroblasts as modulators of local and systemic cancer metabolism. Cancers 11, https://doi.org/10.3390/ cancers 11050619 (2019).

21. Lau, E. Y. et al. Cancer-associated fibroblasts regulate tumor-initiating cell plasticity in hepatocellular carcinoma through c-Met/FRA1/HEY1 signaling. Cell Rep. 15, 1175-1189 (2016).

22. Andriani, F. et al. Conversion to stem-cell state in response to microenvironmental cues is regulated by balance between epithelial and mesenchymal features in lung cancer cells. Mol. Oncol. 10, 253-271 (2016).

23. Penkert, J. et al. On metabolic reprogramming and tumor biology: a comprehensive survey of metabolism in breast cancer. Oncotarget 7, 67626-67649 (2016).

24. Hanahan, D. \& Weinberg, R. A. Hallmarks of cancer: the next generation. Cell 144, 646-674 (2011)

25. Semenza, G. L. Regulation of the breast cancer stem cell phenotype by hypoxia-inducible factors. Clin. Sci. 129, 1037-1045 (2015).

26. Schwab, L. P. et al. Hypoxia-inducible factor 1alpha promotes primary tumor growth and tumor-initiating cell activity in breast cancer. Breast Cancer Res. 14 R6 (2012).

27. Mehla, K. \& Singh, P. K. Metabolic regulation of macrophage polarization in cancer. Trends Cancer 5, 822-834 (2019).

28. Bohn, T. et al. Tumor immunoevasion via acidosis-dependent induction of regulatory tumor-associated macrophages. Nat. Immunol. 19, 1319-1329 (2018).

29. Sharma, N. S. et al. Targeting tumor-intrinsic hexosamine biosynthesis sensitizes pancreatic cancer to anti-PD1 therapy. J. Clin. Investig. 130, 451-465 (2020).

30. Dauer, P. et al. Inactivation of cancer-associated-fibroblasts disrupts oncogenic signaling in pancreatic cancer cells and promotes its regression. Cancer Res. 78, 1321-1333 (2018)

31. Apte, M. V. et al. Periacinar stellate shaped cells in rat pancreas: identification, isolation, and culture. Gut 43, 128-133 (1998).

32. Chytil, A., Magnuson, M. A., Wright, C. V. \& Moses, H. L. Conditional inactivation of the TGF-beta type II receptor using Cre:Lox. Genesis 32, 73-75 (2002).

33. Pacchiano, F. et al. Ureido-substituted benzenesulfonamides potently inhibit carbonic anhydrase IX and show antimetastatic activity in a model of breast cancer metastasis. J. Med. Chem. 54, 1896-1902 (2011). 
34. Hu, Y. \& Smyth, G. K. ELDA: extreme limiting dilution analysis for comparing depleted and enriched populations in stem cell and other assays. J. Immunol. Methods 347, 70-78 (2009).

35. Marcato, P., Dean, C. A., Giacomantonio, C. A. \& Lee, P. W. Aldehyde dehydrogenase: its role as a cancer stem cell marker comes down to the specific isoform. Cell Cycle 10, 1378-1384 (2011).

36. Hamada, S., Masamune, A., Yoshida, N., Takikawa, T. \& Shimosegawa, T. IL-6/ STAT3 plays a regulatory role in the interaction between pancreatic stellate cells and cancer cells. Dig. Dis. Sci. 61, 1561-1571 (2016).

37. Holmer, R., Goumas, F. A., Waetzig, G. H., Rose-John, S. \& Kalthoff, H. Interleukin6: a villain in the drama of pancreatic cancer development and progression. Hepatobiliary Pancreat. Dis. Int 13, 371-380 (2014).

38. Razidlo, G. L., Burton, K. M. \& McNiven, M. A. Interleukin-6 promotes pancreatic cancer cell migration by rapidly activating the small GTPase CDC42. J. Biol. Chem. 293, 11143-11153 (2018).

39. Wu, Y. S. et al. Paracrine IL-6 signaling mediates the effects of pancreatic stellate cells on epithelial-mesenchymal transition via Stat3/Nrf2 pathway in pancreatic cancer cells. Biochim. Biophys. Acta Gen. Subj. 1861, 296-306 (2017)

40. Mace, T. A. et al. IL-6 and PD-L1 antibody blockade combination therapy reduces tumour progression in murine models of pancreatic cancer. Gut $\mathbf{6 7}$ 320-332 (2018).

41. Rycaj, K. \& Tang, D. G. Cell-of-origin of cancer versus cancer stem cells: assays and interpretations. Cancer Res. 75, 4003-4011 (2015).

42. Al-Hajj, M., Wicha, M. S., Benito-Hernandez, A., Morrison, S. J. \& Clarke, M. F. Prospective identification of tumorigenic breast cancer cells. Proc. Natl Acad. Sci. USA 100, 3983-3988 (2003).

43. Nagathihalli, N. S. et al. Signal transducer and activator of transcription 3 , mediated remodeling of the tumor microenvironment results in enhanced tumor drug delivery in a mouse model of pancreatic cancer. Gastroenterology 149, 1932-1943.e1939 (2015).

44. Long, K. B. et al. IL6 receptor blockade enhances chemotherapy efficacy in pancreatic ductal adenocarcinoma. Mol. Cancer Ther. 16, 1898-1908 (2017) 